\title{
Correction to: The association of maternal- fetal attachment with smoking and smoking cessation during pregnancy in The FinnBrain Birth Cohort Study
}

Heidi Jussila ${ }^{1,2^{*}}$ (D, Juho Pelto ${ }^{2}$, Riikka Korja ${ }^{2,3}$, Eeva Ekholm ${ }^{2,4}$, Marjukka Pajulo ${ }^{2,5}$, Linnea Karlsson ${ }^{2,6,7}$ and Hasse Karlsson ${ }^{2,8}$

\section{Correction to: BMC Pregnancy Childbirth 20, 741 (2020) https://doi.org/10.1186/s12884-020-03393-x}

Following publication of the original article [1], the authors reported an error in Tables 2 and 3 headings of the two columns are missing during layout processing. The correct tables are given below.

\begin{abstract}
Author details
'Department of Child Psychiatry, Doctoral Programme of Clinical Investigation, University of Turku, Turku, Finland. ${ }^{2}$ Department of Clinical Medicine, FinnBrain Birth Cohort Study, Turku Brain and Mind Center, University of Turku, Lemminkäisenkatu 3, 20014 Turku, Finland. ${ }^{3}$ Department of Psychology, University of Turku, Turku, Finland. ${ }^{4}$ Department of Obstetrics and Gynecology, Turku University Hospital, Turku, Finland. ${ }^{5}$ Department of Child Psychiatry, University of Turku, Turku, Finland. ${ }^{6}$ Centre for Population Health Research, University of Turku and Turku University Hospital, Turku, Finland. ${ }^{7}$ Department of Child Psychiatry, Turku University Hospital and University of Turku, Turku, Finland. ${ }^{8}$ Department of Psychiatry, Turku University Hospital and University of Turku, Turku, Finland.
\end{abstract}

Published online: 10 April 2021

\section{Reference}

1. Jussila H, Pelto J, Korja R, Ekholm E, Pajulo M, Karlsson L, et al. The association of maternal-fetal attachment with smoking and smoking cessation during pregnancy in The FinnBrain Birth Cohort Study. BMC Pregnancy Childbirth. 2020;20(1):741. https://doi.org/10.1186/s12884-020-03393-X.

The original article can be found online at https://doi.org/10.1186/s12884 020-03393-x

* Correspondence: hemhaan@utu.fi

${ }^{1}$ Department of Child Psychiatry, Doctoral Programme of Clinical

Investigation, University of Turku, Turku, Finland

2Department of Clinical Medicine, FinnBrain Birth Cohort Study, Turku Brain and Mind Center, University of Turku, Lemminkäisenkatu 3, 20014 Turku, Finland

Full list of author information is available at the end of the article

C The Author(s). 2021 Open Access This article is licensed under a Creative Commons Attribution 4.0 International License, which permits use, sharing, adaptation, distribution and reproduction in any medium or format, as long as you give appropriate credit to the original author(s) and the source, provide a link to the Creative Commons licence, and indicate if changes were made. The images or other third party material in this article are included in the article's Creative Commons licence, unless indicated otherwise in a credit line to the material. If material is not included in the article's Creative Commons licence and your intended use is not permitted by statutory regulation or exceeds the permitted use, you will need to obtain permission directly from the copyright holder. To view a copy of this licence, visit http://creativecommons.org/licenses/by/4.0/. The Creative Commons Public Domain Dedication waiver (http://creativecommons.org/publicdomain/zero/1.0/) applies to the data made available in this article, unless otherwise stated in a credit line to the data. 
Table 2 The descriptives statistics of MFA and PPD, and the comparisons between the pregnant smokers and the non-smokers

\begin{tabular}{|c|c|c|c|c|c|c|c|c|}
\hline & \multirow{2}{*}{\multicolumn{3}{|c|}{$\begin{array}{l}\text { Non-smokers } \\
N=3086\end{array}$}} & \multirow{2}{*}{\multicolumn{3}{|c|}{$\begin{array}{l}\text { Smokers } \\
N=612\end{array}$}} & \multirow{2}{*}{\multicolumn{2}{|c|}{$\begin{array}{l}\text { Between-groups } \\
\text { difference }\end{array}$}} \\
\hline & & & & & & & & \\
\hline & $n$ & Mean (SD) & Median (range) & $n$ & Mean (SD) & Median (range) & $p^{a}$ & $r$ \\
\hline \multicolumn{9}{|l|}{ 24gwks } \\
\hline MFAS & 2331 & 86.9 (10.6) & $87(51-116)$ & 409 & $88.0(10.0)$ & $88(44-113)$ & 0.074 & 0.03 \\
\hline Role taking & 2336 & 16.7 (2.8) & $17(6-20)$ & 410 & $16.8(2.7)$ & $17(7-20)$ & 0.703 & 0.01 \\
\hline Differentiation & 2340 & $15.4(2.5)$ & $15(6-20)$ & 410 & $15.7(2.4)$ & $16(7-20)$ & $0.032^{*}$ & 0.04 \\
\hline Interacting & 2338 & $16.6(3.1)$ & $17(5-25)$ & 410 & $17.1(3.0)$ & $17(8-24)$ & $0.004^{* *}$ & 0.05 \\
\hline Attributing & 2335 & 18.5 (3.4) & $19(6-30)$ & 409 & 19.0 (3.2) & $19(11-29)$ & $0.006^{* *}$ & 0.05 \\
\hline Giving & 2338 & $19.7(2.5)$ & $20(9-25)$ & 410 & $19.5(2.5)$ & $20(8-25)$ & 0.091 & 0.03 \\
\hline EPDS & 2317 & $4.8(4.1)$ & $4(0-25)$ & 408 & $6.0(4.3)$ & $5(0-23)$ & $<0.001^{* *}$ & 0.11 \\
\hline SCL-90 Anxiety & 2314 & $3.8(4.2)$ & $3(0-30)$ & 408 & $4.7(4.6)$ & $4(0-24)$ & $<0.001^{* *}$ & 0.08 \\
\hline \multicolumn{9}{|l|}{ 34gwks } \\
\hline MFAS & 2188 & $92.0(10.2)$ & $93(52-116)$ & 373 & $93.1(10.2)$ & $93(55-117)$ & 0.137 & 0.03 \\
\hline Role taking & 2202 & $17.1(2.6)$ & $18(7-20)$ & 374 & $17.1(2.5)$ & $18(8-20)$ & 0.886 & 0.00 \\
\hline Differentiation & 2204 & $15.9(2.4)$ & $16(8-20)$ & 374 & $16.2(2.2)$ & $16(10-20)$ & 0.111 & 0.03 \\
\hline Interacting & 2202 & $18.1(3.1)$ & $18(5-25)$ & 374 & $18.5(2.8)$ & $19(10-25)$ & $0.037^{*}$ & 0.04 \\
\hline Attributing & 2198 & $21.1(3.4)$ & $22(9-30)$ & 373 & $21.8(3.4)$ & $22(12-30)$ & $0.001^{* *}$ & 0.07 \\
\hline Giving & 2198 & $19.8(2.4)$ & $20(10-25)$ & 374 & $19.6(2.5)$ & $20(10-25)$ & 0.171 & 0.03 \\
\hline EPDS & 2191 & $4.7(4.0)$ & $4(0-26)$ & 373 & $5.9(4.5)$ & $5(0-23)$ & $<0.001^{* *}$ & 0.10 \\
\hline \multirow[t]{2}{*}{ SCL-90 Anxiety } & 2186 & $3.1(3.8)$ & $2(0-27)$ & 374 & $3.8(4.6)$ & $3(0-33)$ & $0.012^{*}$ & 0.05 \\
\hline & & $n / N$ & $\%$ & & $n / N$ & $\%$ & $X^{2} p$ & Cramer's $\varphi$ \\
\hline 24 gwks EPDS $\geq 10$ & & 299/2317 & $12.9 \%$ & & $73 / 408$ & $17.9 \%$ & $0.007^{* *}$ & 0.05 \\
\hline $\mathbf{3 4} \mathbf{g w k s}$ EPDS $\geq 10$ & & 287/2191 & $13.1 \%$ & & $84 / 373$ & $22.5 \%$ & $<0.001^{* *}$ & 0.09 \\
\hline
\end{tabular}

The estimates are based on the original data, not the imputed dataset

Attrition in the MFAS questionnaire responses was higher among the smoking women in comparison with the non-smokers $(24$ gwks: $33.2 \%$ vs. $24.5 \%, x 2=20.2$, $p<0.001 ; 34$ gwks: $39.1 \%$ vs. $29.1 \%, X^{2}=23.8, p<0.001$, respectively)

MFAS Maternal-Fetal Attachment Scale and the subscales of Maternal-Fetal Attachment Scale: Role taking, Differentiation, Differentiation of self from fetus, Interacting, Interacting with the fetus, Attributing, Attributing characteristics to the fetus and Giving Giving of self, EPDS Edinburgh (Pre) Postnatal Depression Scale, SCL-90 Anxiety Symptom Checklist - 90, the Anxiety Subscale

${ }^{*} p$-value $<.05$, ** $p$-value $<.01$

${ }^{a}$ Mann-Whitney U test 
Table 3 The descriptives statistics of MFA and PPD, and the comparisons between the smokers who quit and the persistent smokers

\begin{tabular}{|c|c|c|c|c|c|c|c|c|}
\hline & \multirow{2}{*}{\multicolumn{3}{|c|}{$\begin{array}{l}\text { Quit smoking } \\
N=354\end{array}$}} & \multirow{2}{*}{\multicolumn{3}{|c|}{$\begin{array}{l}\text { Continued smoking } \\
N=255\end{array}$}} & \multicolumn{2}{|c|}{$\begin{array}{l}\text { Between-groups } \\
\text { difference }\end{array}$} \\
\hline & & & & & & & 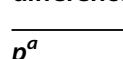 & $r$ \\
\hline \multicolumn{9}{|l|}{24 gwks } \\
\hline MFAS & 257 & $88.6(10.0)$ & $88(57-113)$ & 152 & $87.0(10.0)$ & $87(44-112)$ & 0.102 & 0.08 \\
\hline Role taking & 258 & $16.6(2.7)$ & $17(8-20)$ & 152 & $16.7(2.6)$ & $17(7-20)$ & 0.237 & 0.06 \\
\hline Differentiation & 258 & $15.7(2.4)$ & $16(9-20)$ & 152 & $15.6(2.3)$ & $16(7-20)$ & 0.644 & 0.02 \\
\hline Interacting & 258 & $17.1(3.1)$ & $17(8-24)$ & 152 & $17.0(2.9)$ & $17(9-24)$ & 0.574 & 0.03 \\
\hline Attributing & 257 & $19.1(3.2)$ & $19(11-29)$ & 152 & $19.0(3.2)$ & $19.0(11-29)$ & 0.773 & 0.01 \\
\hline Giving & 258 & $19.8(2.4)$ & $20(13-25)$ & 152 & $18.8(2.6)$ & $19(8-25)$ & $<0.001^{* *}$ & 0.18 \\
\hline EPDS & 256 & $5.6(4.1)$ & $5(0-20)$ & 152 & $6.8(4.5)$ & $6(0-23)$ & $0.006^{* *}$ & 0.14 \\
\hline SCL-90 Anxiety & 256 & $4.3(4.5)$ & $3(0-24)$ & 152 & $5.4(4.7)$ & $4(0-20)$ & $0.007^{* *}$ & 0.13 \\
\hline \multicolumn{9}{|l|}{34 gwks } \\
\hline MFAS & 236 & $93.9(9.7)$ & $93(67-115)$ & 137 & $91.8(10.9)$ & $91(55-117)$ & 0.093 & 0.09 \\
\hline Role taking & 236 & $17.3(2.5)$ & $18(8-20)$ & 138 & $17.0(2.5)$ & $17(8-20)$ & 0.136 & 0.08 \\
\hline Differentiation & 236 & $16.2(2.3)$ & $16(10-20)$ & 138 & $16.1(2.2)$ & $16(10-20)$ & 0.883 & 0.01 \\
\hline Interacting & 236 & $18.5(2.7)$ & $19(12-25)$ & 138 & $18.4(3.0)$ & $18(10-25)$ & 0.555 & 0.03 \\
\hline Attributing & 236 & $21.9(3.3)$ & $22(12-28)$ & 137 & $21.6(3.7)$ & $22(12-30)$ & 0.542 & 0.03 \\
\hline Giving & 236 & $20.1(2.5)$ & $20(12-25)$ & 138 & $18.8(2.5)$ & $18(10-25)$ & $<0.001^{* *}$ & 0.26 \\
\hline EPDS & 235 & $5.4(4.2)$ & $5(0-20)$ & 138 & $6.8(4.9)$ & $6(0-23)$ & $0.007^{* *}$ & 0.14 \\
\hline \multirow[t]{2}{*}{ SCL-90 Anxiety } & 235 & $3.5(4.5)$ & $2(0-33)$ & 139 & $4.5(4.7)$ & $3(0-26)$ & $0.016^{*}$ & 0.12 \\
\hline & & $n / N$ & $\%$ & & $n / N$ & $\%$ & $X^{2} p$ & Cramer's $\varphi$ \\
\hline $\mathbf{2 4} \mathbf{g} \mathbf{w k s} E P D S \geq 10$ & & $36 / 256$ & $14.1 \%$ & & $37 / 152$ & $24.3 \%$ & $0.009^{* *}$ & 0.13 \\
\hline $\mathbf{3 4} \mathbf{g w k s}$ EPDS $\geq 10$ & & $42 / 235$ & $17.9 \%$ & & $42 / 138$ & $30.4 \%$ & $0.005^{* *}$ & 0.15 \\
\hline
\end{tabular}

The estimates are based on the original data, not the imputed dataset

Attrition in the MFAS questionnaire responses was higher among the persistent smokers in comparison with the women who quit smoking during pregnancy (24gwks: $40.9 \%$ vs. $26.0, \mathrm{X} 2=14.5, p<0.001 ; 34$ gwks: $46.8 \%$ vs. $29.6 \%, X 2=18.2, p<0.001$, respectively)

MFAS Maternal-Fetal Attachment Scale and the subscales of Maternal-Fetal Attachment Scale: Role taking, Differentiation Differentiation of self from fetus, Interacting Interacting with the fetus, Attributing Attributing characteristics to the fetus and Giving Giving of self, EPDS Edinburgh (Pre) Postnatal Depression Scale, SCL-90 Anxiety Symptom Checklist - 90, the Anxiety Subscale

${ }^{*} p$-value $<.05,{ }^{* *} p$-value $<.01$

${ }^{\text {a }}$ Mann-Whitney U test 